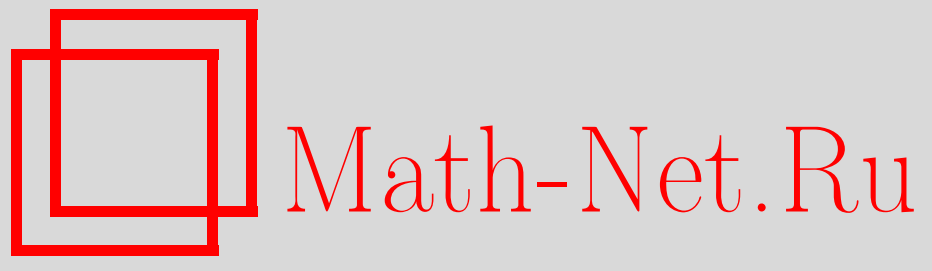

Ф. Гетце, А. А. Наумов, А. Н. Тихомиров, Предельные теоремы для двух классов случайных матриц с зависимыми элементами, Теория вероятн. и ее примен., 2014, том 59, выпуск 1, 61-80

DOI: https://doi.org/10.4213/tvp4550

Использование Общероссийского математического портала Math-Net.Ru подразумевает, что вы прочитали и согласны с пользовательским соглашением http://www . mathnet.ru/rus/agreement

Параметры загрузки:

IP : 52.87 .193 .239

26 апреля 2023 г., 12:16:15

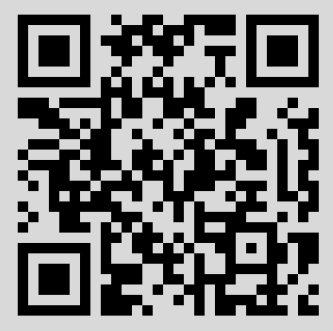




\title{
ПРЕДЕЛЬНЫЕ ТЕОРЕМЫ ДЛЯ ДВУХ КЛАССОВ СЛУЧАЙНЫХ МАТРИЦ С ЗАВИСИМЫМИ ЭЛЕМЕНТАМИ
}

\begin{abstract}
В работе рассматриваются случайные симметричные матрицы с зависимыми элементами. Предположим, что элементы матрицы имеют нулевое математическое ожидание и конечные дисперсии, которые могут быть различными числами. Предполагая выполнение условия Линдеберга и сходимость нормированных сумм дисперсий в каждой строке и столбце к единице, мы доказываем, что ожидаемая эмпирическая спектральная функция распределения собственных значений матрицы сходится к полукруговому закону Вигнера. Результат может быть обобщен на класс ковариационных матриц с зависимыми элементами. В этом случае ожидаемая эмпирическая спектральная функция распределения сходится к закону Марченко-Пастура.
\end{abstract}

Ключевые слова и фразы: случайные матрицы, полукруговой закон, закон Марченко-Пастура, числа Каталана.

1. Введение. Рассмотрим треугольный массив случайных величин $X_{j k}, 1 \leqslant j \leqslant k<\infty$, и предположим, что $\mathbb{E} X_{j k}=0, \mathbb{E} X_{j k}^{2}=\sigma_{j k}^{2}$, и пусть $X_{j k}=X_{k j}$ для всех $1 \leqslant j<k<\infty$. Зафиксируем произвольное $n \in \mathbb{N}$ и сформируем случайную матрицу

$$
\mathbf{X}_{n}=\left\{X_{j k}\right\}_{j, k=1}^{n}
$$

${ }^{*}$ Faculty of Mathematics, University of Bielefeld, 33501 Bielefeld, 1, Germany; e-mail: goetze@math.uni-bielefeld.de

** Московский государственный университет им. М. В. Ломоносова, Москва, Россия; e-mail: naumovne@gmail.com

*** Коми научный центр УРО РАН, Сыктывкарский государственный университет, Сактывкар, Республика Коми, Россия; e-mail: sasha-tikh@yandex.ru

1) Работа поддержана грантом CRC 701 «Spectral Structures and Topological Methods in Mathematics», Bielefeld. Тихомиров А.Н. и Наумов А. А. поддержаны также РФФИ (грант № 14-01-00500). Наумов А. А. поддержан также грантом фонда German Research Foundation (DFG) в рамках International Research Training Group IRTG 1132 и Фондом Саймонса. Тихомиров А. Н. также поддержан программой фундаментальных исследований УрО РАН № 12-Р-1-1013. 
Обозначим через $\lambda_{1} \leqslant \cdots \leqslant \lambda_{n}$ собственные значения матрицы $n^{-1 / 2} \mathbf{X}_{n}$ и определим эмпирическую спектральную функцию распределения

$$
\mathscr{F}^{\mathbf{X}_{n}}(x)=\frac{1}{n} \sum_{i=1}^{n} \mathbb{I}\left(\lambda_{i} \leqslant x\right),
$$

где $\mathbb{I}(B)$ обозначает индикатор события $B$. Дополнительно определим ожидаемую эмпирическую спектральную функцию распределения $F^{\mathbf{X}_{n}}(x):=\mathbb{E} \mathscr{F}^{\mathbf{X}_{n}}(x)$.

Пусть $g(x)$ и $G(x)$ обозначают плотность и функцию распределения стандартного полукругового закона:

$$
g(x)=\frac{1}{2 \pi} \sqrt{4-x^{2}} \mathbb{I}(|x| \leqslant 2), \quad G(x)=\int_{-\infty}^{x} g(u) d u .
$$

Вигнер в работе [1] рассмотрел случай, когда $X_{j k}$ являются независимыми одинаково распределенными случайными величинами и имеют моменты всех порядков (гауссовский случай и симметричный бернуллиевский случай). Он показал, что $F_{n}$ сходится к $G(x)$. Этот результат позже был назван полукруговым законом Вигнера. Полукруговой закон был обобщен в разных направлениях Арнольдом в работе [2]. Например, была установлена сходимость почти наверное эмпирических спектральных функций распределения, но все также при дополнительных моментных ограничениях на элементы матрицы. Наиболее общие условия сходимости почти наверное к полукруговому закону Вигнера для симметричных матриц, рассмотренных выше, были установлены Пастуpoм [3]. Для всех $\tau>0$ определим отношение Линдеберга для случайных матриц соотношением

$$
L_{n}(\tau):=\frac{1}{n^{2}} \sum_{i, j=1}^{n} \mathbb{E} X_{i j}^{2} \mathbb{I}\left(\left|X_{i j}\right| \geqslant \tau \sqrt{n}\right) .
$$

Пастур показал, что условие Линдеберга является достаточным для справедливости полукругового закона. Из его результата, в частности, следует, что если $X_{j k}, 1 \leqslant j \leqslant k \leqslant n$, являются независимыми одинаково распределенными случайными величинами, имеют нулевое математическое ожидание и единичную дисперсию, то $\mathscr{F}^{\mathbf{X}_{n}}(x)$ сходится почти наверное к полукруговому закону Вигнера. Отметим еще раз, что в работе Пастура требовалось выполнение равенства, что $\mathbb{E} X_{j k}^{2}=1$ для всех элементов матрицы. В ряде работ (см., например, [4] и [5]) авторам удалось в некоторых специальных случаях отказаться от выполнения этого требования.

В настоящей работе нас будут интересовать симметричные случайные матрицы с зависимыми элементами. В работе [6] Гётце и Тихомиров обобщили полукруговой закон на класс случайных матриц со структурой случайного поля. Ниже мы определим ансамбль симметричных 
матриц со структурой случайного поля и получим достаточные условия, аналогичные условиям в мартингальной центральной предельной теореме (см., например, [7]). Подчеркнем, что, следуя [4] и [5], в настоящей работе мы не предполагаем, что все дисперсии слагаемых $X_{j k}$, $1 \leqslant j \leqslant k \leqslant n$, являются одинаковыми.

Определим набор $\sigma$-алгебр

$$
\mathfrak{F}^{(i, j)}:=\sigma\left\{X_{k l}: 1 \leqslant k \leqslant l \leqslant n,(k, l) \neq(i, j)\right\}, \quad 1 \leqslant i \leqslant j \leqslant n .
$$

Предположим, что выполнены следующие условия:

$$
\begin{aligned}
& \mathbb{E}\left(X_{i j} \mid \mathfrak{F}^{(i, j)}\right)=0 ; \\
& \frac{1}{n^{2}} \sum_{i, j=1}^{n} \mathbb{E}\left|\mathbb{E}\left(X_{i j}^{2} \mid \mathfrak{F}^{(i, j)}\right)-\sigma_{i j}^{2}\right| \rightarrow 0 \quad \text { при } n \rightarrow \infty ; \\
& L_{n}(\tau) \rightarrow 0 \quad \text { при } n \rightarrow \infty \text { для любого фиксированного } \tau>0 .
\end{aligned}
$$

Всюду далее мы будем использовать условие (3) не только для матрицы $\mathbf{X}_{n}$, но и для других матриц, заменяя элементы $X_{i j}$ в определении отношения Линдеберга соответствующими элементами этих матриц.

Для всех $1 \leqslant i \leqslant n$ обозначим сумму дисперсий в строке через $B_{i}^{2}:=$ $n^{-1} \sum_{j=1}^{n} \sigma_{i j}^{2}$. Наложим условия на поведение дисперсий $\sigma_{i j}^{2}$ :

$$
\begin{aligned}
& \frac{1}{n} \sum_{i=1}^{n}\left|B_{i}^{2}-1\right| \rightarrow 0 \quad \text { при } n \rightarrow \infty ; \\
& \max _{1 \leqslant i \leqslant n} B_{i} \leqslant C,
\end{aligned}
$$

где $C$ - некоторая абсолютная константа.

3 а м е ч а н и е 1. Легко видеть, что условия (4) и (5) следуют из условия

$$
\max _{1 \leqslant i \leqslant n}\left|B_{i}^{2}-1\right| \rightarrow 0 \quad \text { при } n \rightarrow \infty .
$$

Основным результатом настоящей работы является следующая теорема.

Теорема 1. Пусть случайнье матриць $\mathbf{X}_{n}$ удовлетворяют условиям (1)-(5). Тогда

$$
\sup _{x}\left|F^{\mathbf{X}_{n}}(x)-G(x)\right| \rightarrow 0 \quad \text { npu } n \rightarrow \infty .
$$

Зафиксируем произвольные $1 \leqslant i \leqslant j \leqslant n$. Используя свойства математических ожиданий и условие (1), легко видеть, что для всех $(k, l) \neq(i, j), 1 \leqslant k \leqslant l \leqslant n$,

$$
\mathbb{E} X_{i j} X_{k l}=\mathbb{E} \mathbb{E}\left(X_{i j} X_{k l} \mid \mathfrak{F}^{(i, j)}\right)=\mathbb{E} X_{k l} \mathbb{E}\left(X_{i j} \mid \mathfrak{F}^{(i, j)}\right)=0 .
$$


Следовательно, элементы матрицы $\mathbf{X}_{n}$ некоррелированы. Если дополнительно потребовать, чтобы элементы матрицы $\mathbf{X}_{n}$ были независимыми случайными величинами, то условия (1) и (2) будут автоматически выполнены. Следующая теорема вытекает непосредственно из теоремы 1 и представляет собой обобщение полукругового закона для симметричных случайных матриц с независимыми элементами.

Следствие 1. Пусть әлементы $X_{j k}$ случайной матриџь $\mathbf{X}_{n}$ независимы для всех $1 \leqslant j \leqslant k \leqslant n u \mathbb{E} X_{j k}=0, \mathbb{E} X_{j k}^{2}=\sigma_{j k}^{2}$. Предположим, что $\mathbf{X}_{n}$ удовлетворяет условиям (3)-(5). Тогда

$$
\sup _{x}\left|F^{\mathbf{X}_{n}}(x)-G(x)\right| \rightarrow 0 \quad \text { nрu } n \rightarrow \infty .
$$

В работе [6] авторы рассматривают ансамбли симметричных случайных матриц с фиксированным или ограниченным следом. Впервые такие ансамбли были рассмотрены в работах [8] и [9]. Разумным обобщением может служить следующий ансамбль. Предположим, что $X_{j k}$, $1 \leqslant j \leqslant k \leqslant n$, имеют совместное равномерное распределение на многомерном эллипсе или эллипсоиде с полуосями $\sigma_{j k}, 1 \leqslant j \leqslant k \leqslant n$. Это означает, что

$$
\sum_{j=1}^{n} \sum_{k=i}^{n}\left(\frac{X_{j k}}{\sigma_{j k}}\right)^{2}=N
$$

или, в случае эллипсоида,

$$
\sum_{j=1}^{n} \sum_{k=i}^{n}\left(\frac{X_{j k}}{\sigma_{j k}}\right)^{2} \leqslant N
$$

где $N=n(n-1) / 2$. Следуя рассуждениям работы [6], легко установить справедливость условий (1)-(5).

Следствие 2. Пусть элементы $X_{j k}, 1 \leqslant j \leqslant k \leqslant n$, случайной матриць $\mathbf{X}_{n}$ удовлетворяют условиям выие. Тогда

$$
\sup _{x}\left|F^{\mathbf{X}_{n}}(x)-G(x)\right| \rightarrow 0 \quad \text { nрu } n \rightarrow \infty .
$$

Следующий пример демонстрирует, что если условие (4) не выполнено, то последовательность эмпирических спектральных функций распределения может не сходиться к полукруговому закону.

Обозначим через $\mathbf{X}_{n}$ блочную матрицу вида

$$
\mathbf{X}_{n}=\left(\begin{array}{cc}
\mathbf{A} & \mathbf{B} \\
\mathbf{B}^{T} & \mathbf{D}
\end{array}\right)
$$

где матрица $\mathbf{A}$ симметричная и имеет размеры $m \times m$. Матрица $\mathbf{B}$ имеет размеры $m \times(n-m)$, а $\mathbf{D}$ обозначает диагональную матрицу размера 
$(n-m) \times(n-m)$. Предположим, что элементы матриц $\mathbf{A}, \mathbf{B}, \mathbf{D}$ имеют стандартное нормальное распределение. Положим $m:=n / 2$. Легко установить, что условие (4) не выполнено. На рис. 1 изображена компьютерная реализация спектра матрицы $\mathbf{X}_{n}$ в случае $n=2000$.

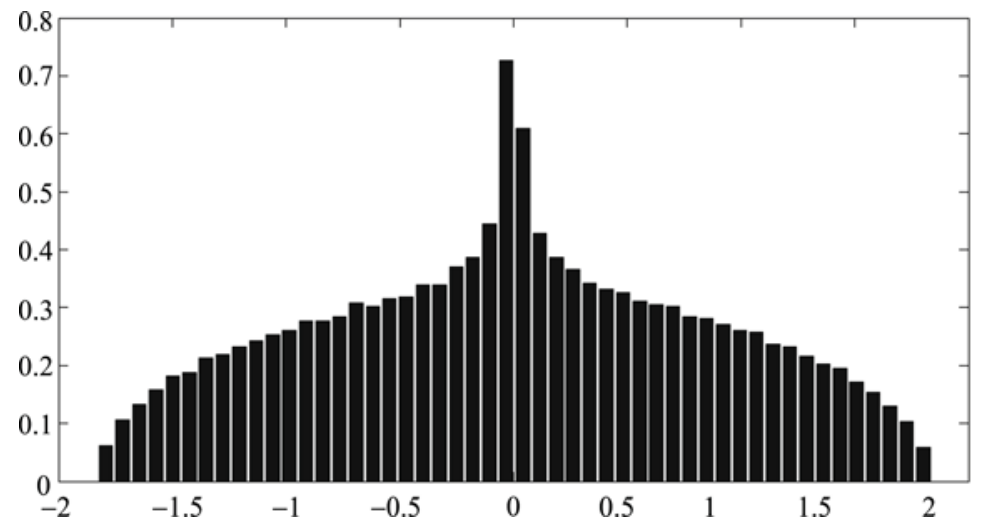

Рис. 1. Спектр случайной матрицы $\mathbf{X}_{n}$.

Определим расстояние Леви между функциями распределения $F_{1}$ и $F_{2}$ формулой

$$
L\left(F_{1}, F_{2}\right)=\inf \left\{\varepsilon>0: F_{1}(x-\varepsilon)-\varepsilon \leqslant F_{2}(x) \leqslant F_{1}(x+\varepsilon)+\varepsilon\right\} .
$$

Следующая теорема иллюстрирует принцип универсальности Линдеберга для случайных матриц.

Теорема 2. Пусть $\mathbf{X}_{n}, \mathbf{Y}_{n}$ - независимые случайные матриџы с $\mathbb{E} X_{j k}=\mathbb{E} Y_{j k}=0 u \mathbb{E} X_{j k}^{2}=\mathbb{E} Y_{j k}^{2}=\sigma_{j k}^{2}$. Предположим, ито матрица $\mathbf{X}_{n}$ удовлетворяет условиям (1)-(4), а матрица $\mathbf{Y}_{n}$ имеет гауссовские независимые әлементы. Дополнительно потребуем, чтобы для матриць $\mathbf{Y}_{n}$ было выполнено условие (3). Тогда

$$
L\left(F^{\mathbf{X}_{n}}(x), F^{\mathbf{Y}_{n}}(x)\right) \rightarrow 0 \quad \text { nрu } n \rightarrow \infty .
$$

В силу теоремы 2 для доказательства теоремы 1 достаточно установить сходимость к полукруговому закону в гауссовском случае.

Теорема 3. Пусть элементы $Y_{j k}$ случайной матрииь $\mathbf{Y}_{n}$ независимь для всех $1 \leqslant j \leqslant k \leqslant n$ и имеют гауссовское распределение с $\mathbb{E} Y_{j k}=0, \mathbb{E} Y_{j k}^{2}=\sigma_{j k}^{2}$. Предположим, ито условия (3)-(5) выполнены. Тогда

$$
\sup _{x}\left|F^{\mathbf{Y}_{n}}(x)-G(x)\right| \rightarrow 0 \quad \text { nрu } n \rightarrow \infty .
$$

3 а м е чан и е 2 . Результат теоремы 3 следует из общего результата работы [4], где используются методы свободной вероятности. Доказательство теоремы 3 , использующее классический метод моментов 
и комбинаторные рассуждения, приведено в работе [10]. Для удобства читателей в настоящей работе мы приведем доказательство теоремы 3 (см. п. 3).

Результат теоремы 1 также может быть обобщен на класс случайных ковариационных матриц. Впервые такие матрицы изучались в работе Уишарта [11]. Рассмотрим массив случайных величин $X_{j k}$, $1 \leqslant j, k<\infty$, таких, что $\mathbb{E} X_{j k}=0$ и $\mathbb{E} X_{j k}^{2}=\sigma_{j k}^{2}$. Сформируем матрицу $\mathbf{X}=\left\{X_{j k}\right\}$ размера $p \times n$. Обозначим через $s_{1}^{2} \leqslant \cdots \leqslant s_{p}^{2}$ собственные значения матрицы $n^{-1} \mathbf{X X}^{*}$ и определим эмпирическую спектральную функцию распределения

$$
\mathscr{F} \mathbf{X X}^{*}(x)=\frac{1}{p} \sum_{i=1}^{p} \mathbb{I}\left(s_{i}^{2} \leqslant x\right) .
$$

Положим $F^{\mathbf{X X}} \mathbf{X}^{*}(x):=\mathbb{E} \mathscr{F} \mathbf{X X}^{*}(x)$. Предположим, что $p=p(n)$ и $\lim _{n \rightarrow \infty} p / n=y$. Без потери общности мы можем считать, что $y \in(0,1]$.

Пусть $g_{y}(x)$ и $G_{y}(x)$ обозначают плотность и функцию распределения закона Марченко-Пастура:

$$
g_{y}(x)=\frac{1}{2 y x \pi} \sqrt{(x-a)(b-x)} \mathbb{I}_{[a, b]}(x), \quad G_{y}(x)=\int_{-\infty}^{x} g_{y}(u) d u,
$$

где $a=(1-\sqrt{y})^{2}, b=(1+\sqrt{y})^{2}$. В работе [12] Марченко и Пастур рассмотрели матрицы $\mathbf{X}$ с независимыми строками и показали, что эмпирическая функция распределения $\mathscr{F}^{\mathbf{X}} \mathbf{X}^{*}(x)$ сходится к $G_{y}(x)$. В частности, из этого результата следует, что если все элементы матрицы $X_{j k}$ являются независимыми одинаково распределенными случайными величинами, то эмпирическая спектральная функция распределения сходится к закону Марченко-Пастура.

Сходимость к закону Марченко-Пастура была обобщена на другие ансамбли случайных матриц (см., например, [13]). Отметим результаты работ [14] и [15]. В частности, в работе [14] Гётце и Тихомиров получили обобщение результата [6] на случай ковариационных матриц и рассмотрели матрицы со структурой случайного поля. В работе [15] Адамчек рассмотрел матрицы со структурой случайного поля, но вместо условия, что все $\mathbb{E} X_{j k}^{2}$ равны 1, предположил выполнение закона больших чисел для сумм квадратов элементов матрицы по строкам и по столбцам.

Мы предположим, что выполнено условие (3), но переопределим $L_{n}(\tau)$ следующим образом:

$$
L_{n}(\tau):=\frac{1}{n p} \sum_{i=1}^{p} \sum_{j=1}^{n} \mathbb{E} X_{i j}^{2} \mathbb{I}\left(\left|X_{i j}\right| \geqslant \tau \sqrt{n}\right) .
$$

Введем набор $\sigma$-алгебр

$$
\mathfrak{F}^{(i, j)}:=\sigma\left\{X_{k l}: 1 \leqslant k \leqslant p, 1 \leqslant l \leqslant n,(k, l) \neq(i, j)\right\},
$$


$1 \leqslant i \leqslant p, 1 \leqslant j \leqslant n$. Будем предполагать, что выполнены следующие условия:

$$
\begin{aligned}
& \mathbb{E}\left(X_{i j} \mid \mathfrak{F}^{(i, j)}\right)=0 ; \\
& \frac{1}{n p} \sum_{i=1}^{p} \sum_{j=1}^{n} \mathbb{E}\left|\mathbb{E}\left(X_{i j}^{2} \mid \mathfrak{F}^{(i, j)}\right)-\sigma_{i j}^{2}\right| \rightarrow 0 \quad \text { при } n \rightarrow \infty ; \\
& L_{n}(\tau) \rightarrow 0 \quad \text { при } n \rightarrow \infty \text { для любого } \tau>0 .
\end{aligned}
$$

Для всех $1 \leqslant i \leqslant p$ обозначим $B_{i}^{2}:=n^{-1} \sum_{k=1}^{n} \sigma_{i k}^{2}$ и для всех $1 \leqslant j \leqslant n$ положим $D_{j}^{2}:=p^{-1} \sum_{l=1}^{p} \sigma_{l j}^{2}$. Наложим условия на поведение $\sigma_{i j}^{2}$ :

$$
\begin{aligned}
& \frac{1}{p} \sum_{i=1}^{p}\left|B_{i}^{2}-1\right| \rightarrow 0 \quad \text { при } n \rightarrow \infty ; \\
& \frac{1}{n} \sum_{j=1}^{n}\left|D_{j}^{2}-1\right| \rightarrow 0 \quad \text { при } n \rightarrow \infty ; \\
& \max \left\{\max _{1 \leqslant i \leqslant p} B_{i}, \max _{1 \leqslant j \leqslant n} D_{j}\right\} \leqslant C,
\end{aligned}
$$

где $C$ - некоторая абсолютная константа.

Теорема 4. Пусть матрица $\mathbf{X}$ удовлетворяет условиям (8)-(13) $u \lim _{n \rightarrow \infty} p / n=y \in(0,1]$. Тогда

$$
\sup _{x}\left|F^{\mathbf{X X}^{*}}(x)-G_{y}(x)\right| \rightarrow 0 \quad \text { nрu } n \rightarrow \infty .
$$

Доказательство теоремы 4 может быть проведено аналогично доказательству теоремы 1 , поэтому мы его опустим.

Всюду далее будем опускать индексы в обозначениях матриц. Под || • || будем понимать спектральную норму матрицы.

2. Доказательство теоремы 2. Пусть $S^{\mathbf{X}}(z)$ и $S^{\mathbf{Y}}(z)$ обозначают преобразования Стилтьеса эмпирических спектральных функций распределения $F^{\mathbf{X}}$ и $F^{\mathbf{Y}}$ соответственно. В силу взаимонепрерывного соответствия между функциями распределения и преобразованиями Стилтьеса, утверждение теоремы 2 будет следовать из соотношения

$$
\left|S^{\mathbf{X}}(z)-S^{\mathbf{Y}}(z)\right| \rightarrow 0 \quad \text { при } n \rightarrow \infty \text { для любого } z \in D,
$$

где $D=\{z \in \mathbb{C}: \operatorname{Im} z>0\}$. Положим

$$
\mathbf{R}^{\mathbf{X}}(z):=\left(\frac{1}{\sqrt{n}} \mathbf{X}-z \mathbf{I}\right)^{-1} \quad \text { и } \quad \mathbf{R}^{\mathbf{Y}}(z):=\left(\frac{1}{\sqrt{n}} \mathbf{Y}-z \mathbf{I}\right)^{-1} .
$$

По определению эмпирической функции распределения

$$
S^{\mathbf{X}}(z)=\frac{1}{n} \operatorname{Tr} \mathbb{E} \mathbf{R}^{\mathbf{X}}(z) \quad \text { и } \quad S^{\mathbf{Y}}(z)=\frac{1}{n} \operatorname{Tr} \mathbb{E} \mathbf{R}^{\mathbf{Y}}(z) .
$$


Заметим, что мы можем заменить произвольное $\tau>0$ в (3) убывающей к нулю последовательностью $\tau_{n}$ такой, что

$$
L_{n}\left(\tau_{n}\right) \rightarrow 0 \text { при } n \rightarrow \infty
$$

и $\lim _{n \rightarrow \infty} \tau_{n} \sqrt{n}=\infty$.

Урезание случайных величин. В этом разделе будет показано, что элементы матриц $\mathbf{X}$ и $\mathbf{Y}$ можно считать равномерно ограниченными по модулю. Мы будем опускать индексы $\mathbf{X}$ и $\mathbf{Y}$ в обозначениях резольвенты и преобразований Стилтьеса.

Рассмотрим произвольную симметричную матрицу $\mathbf{D}$ размера $n \times n$. Положим $\widetilde{\mathbf{X}}=\mathbf{X}+\mathbf{D}$. Пусть

$$
\widetilde{\mathbf{R}}=\left(\frac{1}{\sqrt{n}} \widetilde{\mathbf{X}}-z \mathbf{I}\right)^{-1}, \quad \text { где } z=u+i v .
$$

Лемма 1. Имеет место неравенство

$$
|\operatorname{Tr} \mathbf{R}-\operatorname{Tr} \widetilde{\mathbf{R}}| \leqslant \frac{1}{v^{2}}\left(\operatorname{Tr} \mathbf{D}^{2}\right)^{1 / 2} .
$$

Д о к а з а т е л ь с т в о. В силу соотношения для резольвенты матрицы

$$
\mathbf{R}=\widetilde{\mathbf{R}}-\frac{1}{\sqrt{n}} \mathbf{R D} \widetilde{\mathbf{R}} .
$$

Пусть $z=u+i v, v>0$. Используя оценку для резольвенты матрицы, мы получим

$$
\max \{\|\mathbf{R}\|,\|\widetilde{\mathbf{R}}\|\} \leqslant \frac{1}{v} .
$$

Применяя (17) и (18), легко показать, что

$$
|\operatorname{Tr} \mathbf{R}-\operatorname{Tr} \widetilde{\mathbf{R}}|=\frac{1}{\sqrt{n}}|\operatorname{Tr} \mathbf{R D} \widetilde{\mathbf{R}}| \leqslant \frac{1}{v^{2}}\left(\operatorname{Tr} \mathbf{D}^{2}\right)^{1 / 2} .
$$

Лемма доказана.

Представим элементы матрицы $\mathbf{X}$ в виде $X=\widehat{X}+\check{X}$, где $\widehat{X}:=$ $X \mathbb{I}\left(|X|<\tau_{n} \sqrt{n}\right)$ и $\check{X}:=X \mathbb{I}\left(|X| \geqslant \tau_{n} \sqrt{n}\right)$. Определим матрицу $\widehat{\mathbf{X}}=$ $\left\{\widehat{X}_{i j}\right\}_{i, j=1}^{n}$. Пусть

$$
\widehat{\mathbf{R}}(z):=\left(\frac{1}{\sqrt{n}} \widehat{\mathbf{X}}-z \mathbf{I}\right)^{-1} \quad \text { и } \quad \widehat{S}(z)=\frac{1}{n} \mathbb{E} \operatorname{Tr} \widehat{\mathbf{R}}(z) .
$$

В силу леммы 1

$$
|S(z)-\widehat{S}(z)| \leqslant \frac{1}{v^{2}}\left(\frac{1}{n^{2}} \sum_{i, j=1}^{n} \mathbb{E} X_{i j}^{2} \mathbb{I}\left(\left|X_{i j}\right| \geqslant \tau_{n} \sqrt{n}\right)\right)^{1 / 2}=v^{-2} L_{n}^{1 / 2}\left(\tau_{n}\right) .
$$


Из (16) следует, что

$$
|S(z)-\widehat{S}(z)| \rightarrow 0 \quad \text { при } n \rightarrow \infty .
$$

Введем центрированные случайные величины $\bar{X}_{i j}=\widehat{X}_{i j}-\mathbb{E}\left(\widehat{X}_{i j} \mid \mathfrak{F}^{(i, j)}\right)$ и определим матрицу $\overline{\mathbf{X}}=\left\{\bar{X}_{i j}\right\}_{i, j=1}^{n}$. Пусть

$$
\overline{\mathbf{R}}(z):=\left(\frac{1}{\sqrt{n}} \overline{\mathbf{X}}-z \mathbf{I}\right)^{-1} \text { и } \bar{S}(z)=\frac{1}{n} \mathbb{E} \operatorname{Tr} \overline{\mathbf{R}}(z) .
$$

Снова в силу леммы 1

$$
|\widehat{S}(z)-\bar{S}(z)| \leqslant \frac{1}{v^{2}}\left(\frac{1}{n^{2}} \sum_{i, j=1}^{n} \mathbb{E} X_{i j}^{2} \mathbb{I}\left(\left|X_{i j}\right| \geqslant \tau_{n} \sqrt{n}\right)\right)^{1 / 2}=v^{-2} L_{n}^{1 / 2}\left(\tau_{n}\right) .
$$

Ввиду (16) правая часть стремится к нулю при $n \rightarrow \infty$.

Нам осталось проверить, что в условиях (2) и (4) можно заменить матрицу $\mathbf{X}$ на матрицу $\overline{\mathbf{X}}$. Для всех $1 \leqslant i \leqslant j \leqslant n$

$$
\begin{aligned}
\mathbb{E}\left(\bar{X}_{i j}^{2} \mid \mathfrak{F}^{(i, j)}\right)-\sigma_{i j}^{2}= & \mathbb{E}\left(\bar{X}_{i j}^{2} \mid \mathfrak{F}^{(i, j)}\right)-\mathbb{E}\left(\widehat{X}_{i j}^{2} \mid \mathfrak{F}^{(i, j)}\right) \\
& -\mathbb{E}\left(\check{X}_{i j}^{2} \mid \mathfrak{F}^{(i, j)}\right)+\mathbb{E}\left(X_{i j}^{2} \mid \mathfrak{F}^{(i, j)}\right)-\sigma_{i j}^{2}
\end{aligned}
$$

В силу неравенства треугольника и условий (2), (16)

$$
\begin{aligned}
& \frac{1}{n^{2}} \sum_{i, j=1}^{n} \mathbb{E}\left|\mathbb{E}\left(\bar{X}_{i j}^{2} \mid \mathfrak{F}^{(i, j)}\right)-\sigma_{i j}^{2}\right| \\
& \quad \leqslant \frac{1}{n^{2}} \sum_{i, j=1}^{n} \mathbb{E}\left|\mathbb{E}\left(X_{i j}^{2} \mid \mathfrak{F}^{(i, j)}\right)-\sigma_{i j}^{2}\right|+2 L_{n}\left(\tau_{n}\right) \rightarrow 0 \quad \text { при } n \rightarrow \infty .
\end{aligned}
$$

Используя (19), легко проверить справедливость условия (4) для матрицы $\overline{\mathbf{X}}$.

Определим случайные величины $\widehat{Y}_{i j}=Y_{i j} \mathbb{I}\left(\left|Y_{i j}\right| \leqslant \tau_{n} \sqrt{n}\right)$. Аналогично предыдущим выкладкам можно показать, что мы можем заменить исходную матрицу $\mathbf{Y}$ на матрицу $\widehat{\mathbf{Y}}=\left\{\widehat{Y}_{i j}\right\}_{i, j=1}^{n}$ и

$$
\frac{1}{n^{2}} \sum_{i, j=1}^{n}\left|\mathbb{E} \widehat{Y}_{i j}^{2}-\sigma_{i j}^{2}\right| \rightarrow 0 \quad \text { при } n \rightarrow \infty .
$$

Всюду далее будем предполагать, что $\left|X_{i j}\right| \leqslant \tau_{n} \sqrt{n},\left|Y_{i j}\right| \leqslant \tau_{n} \sqrt{n}$ и выполнены условия (1)-(4).

Универсальность спектра собственных значений. Для доказательства соотношения (14) мы будем использовать метод из работы Бенткуса [16] (см. также [17]). Определим семейство матриц 
$\mathbf{Z}:=\mathbf{Z}(\varphi):=\mathbf{X} \cos \varphi+\mathbf{Y} \sin \varphi$. Легко видеть, что $\mathbf{Z}(0)=\mathbf{X}$ и $\mathbf{Z}(\pi / 2)=\mathbf{Y}$. Положим $\mathbf{W}:=\mathbf{W}(\varphi):=n^{-1 / 2} \mathbf{Z}$ и

$$
\mathbf{R}(z, \varphi):=(\mathbf{W}-z \mathbf{I})^{-1} .
$$

Определим преобразование Стилтьеса

$$
S(z, \varphi):=\frac{1}{n} \sum_{i=1}^{n} \mathbb{E}[\mathbf{R}(z, \varphi)]_{i i} .
$$

Заметим, что $S(z, 0)$ и $S(z, \pi / 2)$ являются преобразованиями Стилтьеса $S^{\mathbf{X}}(z)$ и $S^{\mathbf{Y}}(z)$ соответственно.

В силу теоремы Ньютона-Лейбница

$$
S\left(z, \frac{\pi}{2}\right)-S(z, 0)=\int_{0}^{\pi / 2} \frac{\partial S(z, \varphi)}{\partial \varphi} d \varphi .
$$

Для упрощения записи мы будем опускать аргументы в обозначениях матриц и преобразований Стилтьеса.

Беря производную матрицы $\mathbf{W}$ по аргументу $\theta$, получим

$$
\frac{\partial \mathbf{W}}{\partial \varphi}=\frac{1}{\sqrt{n}} \sum_{i=1}^{n} \sum_{j=1}^{n} \frac{\partial Z_{i j}}{\partial \varphi} \mathbf{e}_{i} \mathbf{e}_{j}^{T},
$$

где $\mathbf{e}_{i}$ обозначает единичный вектор-столбец с 1 на $i$-м месте. Мы можем переписать производную под интегралом в (22) в следующем виде:

$$
\begin{aligned}
\frac{\partial S}{\partial \varphi} & =-\frac{1}{n} \mathbb{E} \operatorname{Tr} \mathbf{R} \frac{\partial \mathbf{W}}{\partial \varphi} \mathbf{R}=-\frac{1}{n^{3 / 2}} \sum_{i=1}^{n} \sum_{j=1}^{n} \mathbb{E} \operatorname{Tr} \mathbf{R} \frac{\partial Z_{i j}}{\partial \varphi} \mathbf{e}_{i} \mathbf{e}_{j}^{T} \mathbf{R} \\
& =\frac{1}{n^{3 / 2}} \sum_{i=1}^{n} \sum_{j=1}^{n} \mathbb{E} \frac{\partial Z_{i j}}{\partial \varphi} u_{i j},
\end{aligned}
$$

где $u_{i j}=-\left[\mathbf{R}^{2}\right]_{j i}$.

Для всех $1 \leqslant i \leqslant j \leqslant n$ определим случайные величины

$$
\xi_{i j}:=Z_{i j}, \quad \widehat{\xi}_{i j}:=\frac{\partial Z_{i j}}{\partial \varphi}=-X_{i j} \sin \varphi+Y_{i j} \cos \varphi,
$$

и наборы случайных величин

$$
\xi^{(i j)}:=\left\{\xi_{k l}: 1 \leqslant k \leqslant l \leqslant n,(k, l) \neq(i, j)\right\} .
$$

Используя формулу Тейлора, можно написать

$$
\begin{aligned}
u_{i j}\left(\xi_{i j}, \xi^{(i j)}\right)= & u_{i j}\left(0, \xi^{(i j)}\right)+\xi_{i j} \frac{\partial u_{i j}}{\partial \xi_{i j}}\left(0, \xi^{(i j)}\right) \\
& +\mathbb{E}_{\theta}(1-\theta) \xi_{i j}^{2} \frac{\partial^{2} u_{i j}}{\partial \xi_{i j}^{2}}\left(\theta \xi_{i j}, \xi^{(i j)}\right),
\end{aligned}
$$


где $\theta$ имеет равномерное распределение на $[0,1]$ и не зависит от $\left(\xi_{i j}, \xi^{(i j)}\right)$. Умножая обе части последнего равенства на $\hat{\xi}_{i j}$ и переходя к математическим ожиданиям, получим

$$
\begin{aligned}
\mathbb{E} \widehat{\xi}_{i j} u_{i j}\left(\xi_{i j}, \xi^{(i j)}\right)= & \mathbb{E} \widehat{\xi}_{i j} u_{i j}\left(0, \xi^{(i j)}\right)+\mathbb{E} \widehat{\xi}_{i j} \xi_{i j} \frac{\partial u_{i j}}{\partial \xi_{i j}}\left(0, \xi^{(i j)}\right) \\
& +\mathbb{E}(1-\theta) \widehat{\xi}_{i j} \xi_{i j}^{2} \frac{\partial^{2} u_{i j}}{\partial \xi_{i j}^{2}}\left(\theta \xi_{i j}, \xi^{(i j)}\right)
\end{aligned}
$$

В силу независимости $Y_{i j}$ и $\xi^{(i j)}$ имеем

$$
\mathbb{E} Y_{i j} u_{i j}\left(0, \xi^{(i j)}\right)=\mathbb{E} Y_{i j} \mathbb{E} u_{i j}\left(0, \xi^{(i j)}\right)=0
$$

По свойству условного математического ожидания и в силу условия (1)

$$
\mathbb{E} X_{i j} u_{i j}\left(0, \xi^{(i j)}\right)=\mathbb{E} u_{i j}\left(0, \xi^{(i j)}\right) \mathbb{E}\left(X_{i j} \mid \mathfrak{F}^{(i, j)}\right)=0 .
$$

Используя (24), (25) и (26), мы можем переписать (23) следующим обра3ом:

$$
\begin{aligned}
\frac{\partial S}{\partial \varphi}= & \frac{1}{n^{2}} \sum_{i, j=1}^{n} \mathbb{E} \widehat{\xi}_{i j} \xi_{i j} \frac{\partial u_{i j}}{\partial \xi_{i j}}\left(0, \xi^{(i j)}\right) \\
& +\frac{1}{n^{2}} \sum_{i, j=1}^{n} \mathbb{E}(1-\theta) \widehat{\xi}_{i j} \xi_{i j}^{2} \frac{\partial^{2} u_{i j}}{\partial \xi_{i j}^{2}}\left(\theta \xi_{i j}, \xi^{(i j)}\right)=: \mathbb{A}_{1}+\mathbb{A}_{2}
\end{aligned}
$$

Легко видеть, что

$$
\widehat{\xi}_{i j} \xi_{i j}=-\frac{1}{2} X_{i j}^{2} \sin 2 \varphi+X_{i j} Y_{i j} \cos ^{2} \varphi-X_{i j} Y_{i j} \sin ^{2} \varphi+\frac{1}{2} \varphi Y_{i j}^{2} \sin 2
$$

Случайные величины $Y_{i j}$ не зависят от $X_{i j}$ и $\xi^{(i j)}$. Используя этот факт, мы можем написать

$$
\begin{aligned}
\mathbb{E} X_{i j} Y_{i j} \frac{\partial u_{i j}}{\partial \xi_{i j}}\left(0, \xi^{(i j)}\right) & =\mathbb{E} Y_{i j} \mathbb{E} X_{i j} \frac{\partial u_{i j}}{\partial \xi_{i j}}\left(0, \xi^{(i j)}\right)=0 \\
\mathbb{E} Y_{i j}^{2} \frac{\partial u_{i j}}{\partial \xi_{i j}}\left(0, \xi^{(i j)}\right) & =\sigma_{i j}^{2} \mathbb{E} \frac{\partial u_{i j}}{\partial \xi_{i j}}\left(0, \xi^{(i j)}\right)
\end{aligned}
$$

Снова в силу свойства условного математического ожидания получим

$$
\mathbb{E} X_{i j}^{2} \frac{\partial u_{i j}}{\partial \xi_{i j}}\left(0, \xi^{(i j)}\right)=\mathbb{E} \frac{\partial u_{i j}}{\partial \xi_{i j}}\left(0, \xi^{(i j)}\right) \mathbb{E}\left(X_{i j}^{2} \mid \mathfrak{F}^{(i, j)}\right)
$$

Легко установить, что производная $u_{i j}=-\left[\mathbf{R}^{2}\right]_{j i}$ равна

$$
\frac{\partial u_{i j}}{\partial \xi_{i j}}=\left[\mathbf{R}^{2} \frac{\partial \mathbf{Z}}{\partial \xi_{i j}} \mathbf{R}\right]_{j i}+\left[\mathbf{R} \frac{\partial \mathbf{Z}}{\partial \xi_{i j}} \mathbf{R}^{2}\right]_{j i}
$$




$$
\begin{aligned}
= & \frac{1}{\sqrt{n}}\left[\mathbf{R}^{2} \mathbf{e}_{i} \mathbf{e}_{j}^{T} \mathbf{R}\right]_{j i}+\frac{1}{\sqrt{n}}\left[\mathbf{R}^{2} \mathbf{e}_{j} \mathbf{e}_{i}^{T} \mathbf{R}\right]_{j i} \\
& +\frac{1}{\sqrt{n}}\left[\mathbf{R e}_{i} \mathbf{e}_{j}^{T} \mathbf{R}^{2}\right]_{j i}+\frac{1}{\sqrt{n}}\left[\mathbf{R e}_{j} \mathbf{e}_{i}^{T} \mathbf{R}^{2}\right]_{j i} \\
= & \frac{1}{\sqrt{n}}\left[\mathbf{R}^{2}\right]_{j i}[\mathbf{R}]_{j i}+\frac{1}{\sqrt{n}}\left[\mathbf{R}^{2}\right]_{j j}[\mathbf{R}]_{i i}+\frac{1}{\sqrt{n}}[\mathbf{R}]_{j i}\left[\mathbf{R}^{2}\right]_{j i}+\frac{1}{\sqrt{n}}[\mathbf{R}]_{j j}\left[\mathbf{R}^{2}\right]_{i i} .
\end{aligned}
$$

Используя очевидную оценку резольвенты матрицы, $\|\mathbf{R}\| \leqslant v^{-1}$, получим

$$
\left|\frac{\partial u_{i j}}{\partial \xi_{i j}}\right| \leqslant \frac{C}{\sqrt{n} v^{3}} .
$$

Из (27)-(30) и (19), (20) можем заключить, что

$$
\left|\mathbb{A}_{1}\right| \leqslant \frac{C}{n^{2} v^{3}} \sum_{i, j=1}^{n} \mathbb{E}\left|\mathbb{E}\left(X_{i j}^{2} \mid \mathfrak{F}^{(i, j)}\right)-\sigma_{i j}^{2}\right| \rightarrow 0 \quad \text { при } n \rightarrow \infty .
$$

Нам остается оценить $\mathbb{A}_{2}$. Вычислим вторую производную функции $u_{i j}$ :

$$
\begin{aligned}
\frac{\partial^{2} u_{i j}}{\partial \xi_{i j}^{2}}= & -\left[\mathbf{R}^{2} \frac{\partial \mathbf{V}}{\partial \xi_{i j}} \mathbf{R} \frac{\partial \mathbf{V}}{\partial \xi_{i j}} \mathbf{R}\right]_{j i}-\left[\mathbf{R} \frac{\partial \mathbf{V}}{\partial \xi_{i j}} \mathbf{R}^{2} \frac{\partial \mathbf{V}}{\partial \xi_{i j}} \mathbf{R}\right]_{j i} \\
& -\left[\mathbf{R} \frac{\partial \mathbf{V}}{\partial \xi_{i j}} \mathbf{R} \frac{\partial \mathbf{V}}{\partial \xi_{i j}} \mathbf{R}^{2}\right]_{j i}=: \mathbb{T}_{1}+\mathbb{T}_{2}+\mathbb{T}_{3} .
\end{aligned}
$$

Представим член $\mathbb{T}_{1}$ в виде суммы:

$$
\mathbb{T}_{1}=-\left[\mathbf{R}^{2} \frac{\partial \mathbf{V}}{\partial \xi_{i j}} \mathbf{R} \frac{\partial \mathbf{V}}{\partial \xi_{i j}} \mathbf{R}\right]_{j i}=: \mathbb{T}_{11}+\mathbb{T}_{12}+\mathbb{T}_{13}+\mathbb{T}_{14},
$$

где мы обозначили

$$
\begin{array}{ll}
\mathbb{T}_{11}=-\frac{1}{n}\left[\mathbf{R}^{2}\right]_{j i}[\mathbf{R}]_{j i}[\mathbf{R}]_{j i}, & \mathbb{T}_{12}=-\frac{1}{n}\left[\mathbf{R}^{2}\right]_{j i}[\mathbf{R}]_{j j}[\mathbf{R}]_{i i}, \\
\mathbb{T}_{13}=-\frac{1}{n}\left[\mathbf{R}^{2}\right]_{j j}[\mathbf{R}]_{i i}[\mathbf{R}]_{j i}, & \mathbb{T}_{14}=-\frac{1}{n}\left[\mathbf{R}^{2}\right]_{j j}[\mathbf{R}]_{i j}[\mathbf{R}]_{i i} .
\end{array}
$$

Снова используя оценку $\|\mathbf{R}\| \leqslant v^{-1}$, можно показать, что

$$
\max \left\{\left|\mathbb{T}_{11}\right|,\left|\mathbb{T}_{12}\right|,\left|\mathbb{T}_{13}\right|,\left|\mathbb{T}_{14}\right|\right\} \leqslant \frac{C}{n v^{4}} .
$$

Из этих оценок и разложения (32) следует, что $\left|\mathbb{T}_{1}\right| \leqslant C /\left(n v^{4}\right)$. Повторяя рассуждения выше, можно показать, что $\max \left\{\left|\mathbb{T}_{2}\right|,\left|\mathbb{T}_{3}\right|\right\} \leqslant C /\left(n v^{4}\right)$. Окончательно имеем

$$
\left|\frac{\partial^{2} u_{i j}}{\partial \xi_{i j}^{2}}\left(\theta \xi_{i j}, \xi^{(i j)}\right)\right| \leqslant \frac{C}{n v^{4}} .
$$

Используя предположения $\left|\xi_{i j}\right| \leqslant \tau_{n} \sqrt{n}$ и (4), получим оценку

$$
\left|\mathbb{A}_{2}\right| \leqslant \frac{C \tau_{n}}{v^{4}} .
$$

Устремляя $\tau_{n}$ к нулю, можно вывести справедливость утверждения теоремы 2 из (22), (23), (31) и (33). 
3. Доказательство теоремы 3. Для доказательства теоремы мы будем использовать метод моментов. Легко видеть, что моменты ожидаемой эмпирической функции распределения $F^{\mathbf{Y}}(x)$ могут быть переписаны в виде нормированного следа степени матрицы $\mathbf{Y}$ :

$$
\int_{\mathbb{R}} x^{k} d F^{\mathbf{Y}}(x)=\mathbb{E} \frac{1}{n} \operatorname{Tr}\left(\frac{1}{\sqrt{n}} \mathbf{Y}\right)^{k} .
$$

Для доказательства сходимости последовательности $F^{\mathbf{Y}}(x)$ к $G(x)$ достаточно показать, что

$$
\mathbb{E} \frac{1}{n} \operatorname{Tr}\left(\frac{1}{\sqrt{n}} \mathbf{Y}\right)^{k}=\int_{\mathbb{R}} x^{k} d G(x)+o_{k}(1)
$$

для всех $k \geqslant 1$, где $o_{k}(1)$ стремится к нулю при $n \rightarrow \infty$ для любого фиксированного $k$.

Хорошо известно, что моменты полукругового закона равны

$$
\beta_{k}=\int_{\mathbb{R}} x^{k} d G(x)= \begin{cases}\frac{1}{m+1}\left(\begin{array}{c}
2 m \\
m
\end{array}\right), & k=2 m, \\
0, & k=2 m+1 .\end{cases}
$$

Отметим, что моменты $\beta_{2 m}$ совпадают с числами Каталана.

Всюду далее в этом пункте мы будем использовать обозначения и определения из [18]. Графом будем называть триплет $(E, V, F)$, где $E$ множество ребер, $V$ - множество вершин и $F-$ функция, $F: E \rightarrow$ $V \times V$. Пусть $\mathbf{i}=\left(i_{1}, \ldots, i_{k}\right)$ обозначает вектор, принимающий значения из множества $\{1, \ldots, n\}^{k}$. По вектору $\mathbf{i}$ построим Г-граф следующим образом. Нарисуем горизонтальную линию и нанесем на нее числа $i_{1}, \ldots, i_{k}$. Будем рассматривать различные числа в качестве вершин и нарисуем $k$ ребер $e_{j}$ из $i_{j}$ в $i_{j+1}, j=1, \ldots, k$, используя соглашение $i_{k+1}=i_{1}$. Обозначим число различных $i_{j}$ через $t$. Построенный таким образом граф будем обозначать $\Gamma(k, t)$.

Будем говорить, что два $\Gamma(k, t)$-графа изоморфны, если один может быть получен из другого перестановкой $(1, \ldots, n)$. В силу этого определения все Г графы могут быть разбиты на классы изоморфных между собой графов. Назовем $\Gamma(k, t)$-граф каноническим, если он удовлетворяет следующим условиям:

1) множество вершин есть $\{1, \ldots, t\}$;

2) множество ребер есть $\left\{e_{1}, \ldots, e_{k}\right\}$;

3 ) существует функция $g$ из $\{1, \ldots, k\}$ в $\{1, \ldots, t\}$ такая, что $g(1)=1$ и $g(i) \leqslant \max \{g(1), \ldots, g(i-1)\}+1$ для $1<i \leqslant k$;

4) $F\left(e_{i}\right)=(g(i), g(i+1))$, для $i=1, \ldots, k$, где мы используем соглашение $g(k+1)=g(1)=1$. 
Легко видеть, что каждый класс изоморфных графов содержит один и только один канонический Г-граф с ассоциированной с ним функцией $g$. Произвольный граф в этой категории может быть определен с помощью соотношения $F\left(e_{j}\right)=\left(i_{g(j)}, i_{g(j+1)}\right)$. Легко видеть, что число $\Gamma(k, t)$ графов в каждом классе изоморфных графов равно $n(n-1) \cdots(n-t+1)$.

Разобьем все канонические графы на три категории. Категория $C_{1}$ содержит все канонические $\Gamma(k, t)$-графы, у которых каждое ребро совпадает ровно с одним другим ребром, идущим в противоположном направлении, и граф несовпадающих ребер является деревом. Легко видеть, что если $k$ нечетно, то категория $C_{1}$ не содержит ни одного графа. Если $k$ четно, скажем, $k=2 m$, обозначим $\Gamma(k, t)$-граф через $\Gamma_{1}(2 m)$. Категория $C_{2}$ содержит все канонические графы, у которых существует хотя бы одно ребро с нечетной кратностью. Граф из этой категории обозначим через $\Gamma_{2}(k, t)$. Категория $C_{3}$ состоит из всех остальных канонических графов, которые будем обозначать $\Gamma_{3}(k, t)$.

Известно (см. [18, лемма 2.4]), что число канонических $\Gamma_{1}(2 m)$ графов равно

$$
\frac{1}{m+1}\left(\begin{array}{c}
2 m \\
m
\end{array}\right) \text {. }
$$

Перепишем нормированный след степени матрицы $\mathbf{Y}$ в виде

$$
\operatorname{Tr}\left(\frac{1}{\sqrt{n}} \mathbf{Y}\right)^{k}=\frac{1}{n^{k / 2}} \sum_{i_{1}, i_{2}, \ldots, i_{k}} Y_{i_{1} i_{2}} Y_{i_{2} i_{3}} \cdots Y_{i_{k} i_{1}}
$$

где сумма берется по всем векторам $\mathbf{i}=\left(i_{1}, \ldots, i_{k}\right) \in\{1, \ldots, n\}^{k}$.

По каждому вектору i построим граф $G(\mathbf{i})$ как указано выше. Обозначим $Y(\mathbf{i})=Y(G(\mathbf{i}))$.

Мы можем разбить моменты $F^{\mathbf{Y}}(x)$ на три группы:

$$
\mathbb{E} \frac{1}{n} \operatorname{Tr}\left(\frac{1}{\sqrt{n}} \mathbf{Y}\right)^{k}=\frac{1}{n^{k / 2+1}} \sum_{\mathbf{i}} \mathbb{E} Y_{i_{1} i_{2}} Y_{i_{2} i_{3}} \cdots Y_{i_{k} i_{1}}=S_{1}+S_{2}+S_{3},
$$

где

$$
S_{j}=\frac{1}{n^{k / 2+1}} \sum_{\Gamma(k, t) \in C_{j}} \sum_{G(\mathbf{i}) \in \Gamma(k, t)} \mathbb{E} Y(G(\mathbf{i})),
$$

и суммирование в первой сумме $\sum_{\Gamma(k, t) \in C_{j}}$ производится по всем каноническим $\Gamma(k, t)$-графам в категории $C_{j}$, а суммирование в $\sum_{G(\mathbf{i}) \in \Gamma(k, t)}$ производится по всем графам, изоморфным данному каноническому графу.

Из независимости $Y_{i j}$ и равенства $\mathbb{E} Y_{i j}^{2 s-1}=0, s \geqslant 1$, следует, что $S_{2}=0$.

Для графов из категорий $C_{1}$ и $C_{3}$ введем дополнительные обозначения. Рассмотрим $\Gamma(k, t)$-граф $G(\mathbf{i})$. Без потери общности будем считать, что $i_{l}, l=1, \ldots, t$, являются различными координатами вектора 
$\mathbf{i}$, и определим вектор $\mathbf{i}_{t}=\left(i_{1}, \ldots, i_{t}\right)$. Положим $G\left(\mathbf{i}_{t}\right):=G(\mathbf{i})$. Пусть $\widetilde{\imath}_{t}=\left(i_{1}, \ldots, i_{q-1}, i_{q+1}, \ldots, i_{t}\right)$ и $\widehat{\imath}_{t}=\left(i_{1}, \ldots, i_{p-1}, i_{p+1}, \ldots, i_{q-1}, i_{q+1}, \ldots, i_{t}\right)$ обозначают векторы, полученные из $\mathbf{i}_{t}$ удалением элементов в позициях $q$ и $p, q$ соответственно. Дополнительно потребуем, чтобы значения координат $\widehat{\imath}_{t}$ не совпадали с $i_{p}$. Обозначим граф без вершины $i_{q}$ и всех инцидентных ей ребер через $G\left(\widetilde{\imath}_{t}\right)$. Если через вершину $i_{q}$ проходит цикл, то обозначим через $G^{\prime}\left(\mathbf{i}_{t}\right)$ граф без этого цикла. Через $\widetilde{G}\left(\mathbf{i}_{t}\right)$ обозначим граф, полученный из $G\left(\mathbf{i}_{t}\right)$ удалением ребра между $i_{p}$ и $i_{q}$ с учетом кратности.

Теперь оценим $S_{3}$. Для графа из категории $C_{3}$ мы знаем, что $k$ должно быть четным, например, $k=2 m$. Мы изобразили пример $\Gamma_{3}(k, t)$-графа на рис. 2. Этот граф соответствует члену $Y\left(G\left(\mathbf{i}_{3}\right)\right)=$ $Y_{i_{1} i_{1}}^{2} Y_{i_{1} i_{2}}^{2} Y_{i_{2} i_{3}}^{4} Y_{i_{3} i_{3}}^{2}$.

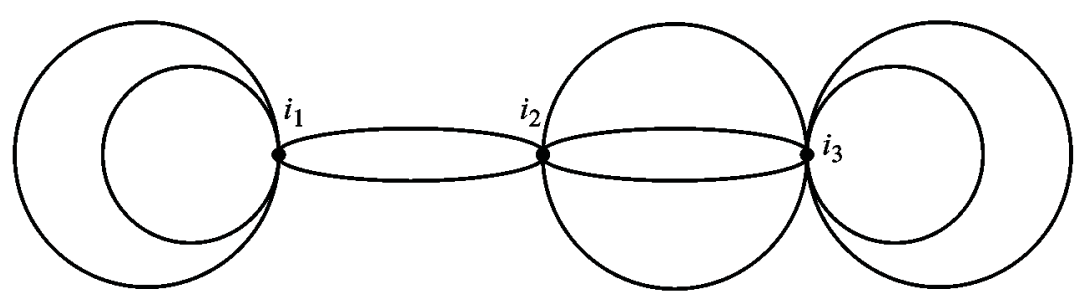

Рис. 2. Граф $\Gamma_{3}(10,3)$.

Заметим, что $\mathbb{E} Y_{i_{p} i_{q}}^{2 s} \leqslant s \sigma_{i_{p} i_{q}}^{2 s}$. Это позволяет нам оценивать моменты высших порядков через дисперсии.

Каждый граф $G$ из категории $C_{3}$ можно представить в виде объединения $G=G_{1} \cup G_{2}$ следующим образом. Раскрасим все ребра $G$ в два цвета, причем совпадающие ребра будут иметь один цвет. Выберем граф $G_{1}$ так, чтобы было выполнено одно из следующих условий:

i) граф $G_{1}$ содержит одну вершину и все инцидентные ей петли;

ii) граф $G_{1}$ содержит две вершины и ребро между ними, причем кратность ребра больше двух;

iii) каждое ребро из $G_{1}$ совпадает ровно с одним ребром, идущим в противоположном направлении, и граф из несовпадающих ребер представляет собой простой цикл.

Предположим, что сумма кратностей всех ребер графа $G_{1}$ равна $2 s$. Нам остается рассмотреть оставшиеся $2(m-s)$ ребер графа $G_{2}$.

Обозначим через $V_{1}, E_{1}$ и $V_{2}, E_{2}$ множества вершин и ребер графов $G_{1}$ и $G_{2}$ соответственно. Мы определим $\mathbf{i}_{t}^{1}:=\left(i_{l}: i_{l} \in V_{1}\right)$. Зафиксируем граф $G_{1}$ и рассмотрим граф $G_{2}$.

a) Если $E_{2}$ пусто, то переходим на шаг с). В противном случае рассмотрим следующие возможности. 
1. Существует петля кратности $2 a, a \geqslant 1$, инцидентная вершине $i_{q} \in V_{2}$. В этом случае мы имеем оценку

$$
\frac{1}{n^{m+1}} \sum_{G(\mathbf{i}) \in \Gamma(2 m, t)} \mathbb{E} Y(G(\mathbf{i})) \leqslant a \frac{1}{n^{m+1}} \sum_{\mathbf{i}_{t}} \mathbb{E}\left[Y\left(G^{\prime}\left(\mathbf{i}_{t}\right)\right)\right] \sigma_{i_{q} i_{q}}^{2 a} .
$$

Применяя $a$ раз неравенство $n^{-1} \sigma_{i_{q} i_{q}}^{2} \leqslant B_{i_{q}}^{2}$ и условие $(5)$, мы удалим все петли, инцидентные вершине $i_{q}$.

2. Не существует петель, инцидентных $i_{q} \in V_{2} \backslash V_{1}$, но $i_{q}$ соединено только с одной вершиной $i_{p} \in V_{2}$ ребром из $G_{2}$ и кратность этого ребра равна $2 b, b \geqslant 1$. В этом случае имеем оценку

$$
\frac{1}{n^{m+1}} \sum_{G(\mathbf{i}) \in \Gamma(2 m, t)} \mathbb{E} Y(G(\mathbf{i})) \leqslant b \frac{1}{n^{m+1}} \sum_{\mathbf{i}_{t}} \mathbb{E}\left[Y\left(G\left(\mathbf{i}_{t}\right)\right)\right] \sum_{i_{q}=1}^{n} \sigma_{i_{p} i_{q}}^{2 b} .
$$

Мы можем использовать $b-1$ раз неравенство $n^{-1} \sigma_{i_{p} i_{q}}^{2} \leqslant B_{i_{p}}^{2}$ и условие (5) и последовательно удалить все ребра за исключением двух. На последнем шаге мы можем снова использовать (5) и удалить $i_{q}$.

3. Не существует петель в $G_{2}$ и не существует вершин в $V_{2} \backslash V_{1}$, которые были бы соединены в точности с одной вершиной из $V_{2}$. Тогда мы можем взять любые две вершины, скажем, $i_{p}$ и $i_{q}$, из $V_{2}$ и написать оценку

$$
\frac{1}{n^{m+1}} \sum_{G(\mathbf{i}) \in \Gamma(2 m, t)} \mathbb{E} Y(G(\mathbf{i})) \leqslant b \frac{1}{n^{m+1}} \sum_{\mathbf{i}_{t}} \mathbb{E}\left[Y\left(\widetilde{G}\left(\mathbf{i}_{t}\right)\right)\right] \sigma_{i_{p} i_{q}}^{2 c} .
$$

Используя $c$ раз неравенство $n^{-1} \sigma_{i_{p} i_{q}}^{2} \leqslant B_{i_{p}}^{2}$ и условие $(5)$, удалим все ребра между $i_{p}$ и $i_{q}$.

b) Переходим на шаг а).

c) Легко видеть, что на каждого шаге а) мы используем одно и то же условие (5). Следовательно, мы можем написать

$$
\frac{1}{n^{m+1}} \sum_{G(\mathbf{i}) \in \Gamma(2 m, t)} \mathbb{E} Y(G(\mathbf{i})) \leqslant{ }_{m} \frac{C^{2(m-s)} n^{m-s}}{n^{m+1}} \sum_{\mathbf{i}_{t}^{1}} \mathbb{E} Y\left(G_{1}\left(\mathbf{i}_{t}^{1}\right)\right) .
$$

Остается оценить правую часть в (35). В случае і) напишем оценку

$$
\begin{aligned}
\frac{1}{n^{s+1}} \sum_{i_{1}=1}^{n} \mathbb{E} Y_{i_{1} i_{1}}^{2 s} & \leqslant s \frac{1}{n^{s+1}} \sum_{i_{1}=1}^{n} \sigma_{i_{1} i_{1}}^{2 s} \leqslant \frac{C^{2(s-1)}}{n^{2}} \sum_{i_{1}=1}^{n} \sigma_{i_{1} i_{1}}^{2} \\
& \leqslant C^{2(s-1)} \tau_{n}^{2}+\frac{C^{2(s-1)}}{n^{2}} \sum_{i_{1}=1}^{n} \mathbb{E} Y_{i_{1} i_{1}}^{2} \mathbb{I}\left(\left|Y_{i_{1} i_{1}}\right| \geqslant \tau_{n} \sqrt{n}\right) \\
& \leqslant C^{2(s-1)} \tau_{n}^{2}+C^{2(s-1)} L_{n}\left(\tau_{n}\right)=o_{s}(1)
\end{aligned}
$$

где мы использовали неравенство $n^{-1} \sigma_{i_{1} i_{1}}^{2} \leqslant B_{i_{1}}^{2}$ и условие $(5)$. 
В случае іi) имеем оценку

$$
\begin{aligned}
& \frac{1}{n^{s+1}} \sum_{\substack{i_{1}, i_{2}=1 \\
i_{1} \neq i_{2}}}^{n} \mathbb{E} Y_{i_{1} i_{2}}^{2 s} \leqslant s \frac{1}{n^{s+1}} \sum_{i_{1}, i_{2}=1}^{n} \sigma_{i_{1} i_{2}}^{2 s} \leqslant \frac{C^{2(s-2)}}{n^{3}} \sum_{i_{1}, i_{2}=1}^{n} \sigma_{i_{1} i_{2}}^{4} \\
& \leqslant C^{2(s-2)} \frac{\tau_{n}^{2}}{n^{2}} \sum_{i_{1}, i_{2}=1}^{n} \sigma_{i_{1} i_{2}}^{2}+\frac{C^{2(s-2)}}{n^{3}} \sum_{i_{1}, i_{2}=1}^{n} \sigma_{i_{1} i_{2}}^{2} \mathbb{E} Y_{i_{1} i_{2}}^{2} \mathbb{I}\left(\left|Y_{i_{1} i_{2}}\right| \geqslant \tau_{n} \sqrt{n}\right) \\
& \leqslant C^{2(s-1)} \tau_{n}^{2}+C^{2(s-1)} L_{n}\left(\tau_{n}\right)=o_{s}(1),
\end{aligned}
$$

где мы снова использовали неравенство $n^{-1} \sigma_{i_{1} i_{1}}^{2} \leqslant B_{i_{1}}^{2}$ и $(5)$.

Остается рассмотреть случай iii). Для этого нам потребуются дополнительные обозначения. Переобозначим вершины из $V_{1}$ и предположим, что $\mathbf{i}_{t}^{1}=\mathbf{i}_{s}:=\left(i_{1}, \ldots, i_{s}\right)$. Через $G_{1}\left(\mathbf{i}_{s}\right)$ обозначим граф $G_{1}\left(\mathbf{i}_{t}^{1}\right)$. Используя введенные ранее обозначения $\widetilde{\imath}_{s}, \widehat{\imath}_{s}$ и $\widetilde{G}_{1}\left(\mathbf{i}_{s}\right)$, будем предполагать, что $p=1$ и $q=2$. Через $\widehat{G}_{1}\left(\widetilde{\imath}_{s}\right)$ обозначим граф, полученный из $\widetilde{G}_{1}\left(\mathbf{i}_{s}\right)$ удалением вершины $i_{2}$ и ребра между $i_{2}$ и $i_{3}$ с учетом кратности. Напишем

$$
\begin{aligned}
& \frac{1}{n^{s+1}} \sum_{\mathbf{i}_{s}} \mathbb{E} Y\left(G_{1}\left(\mathbf{i}_{s}\right)\right) \leqslant \frac{\tau_{n}^{2}}{n^{s}} \sum_{\mathbf{i}_{s}} \mathbb{E} Y\left(\widetilde{G}_{1}\left(\mathbf{i}_{s}\right)\right) \\
& \quad+\frac{1}{n^{s+1}} \sum_{\mathbf{i}_{s}} \mathbb{E} Y\left(\widetilde{G}_{1}\left(\mathbf{i}_{s}\right)\right) \mathbb{E} Y_{i_{1} i_{2}}^{2} \mathbb{I}\left(\left|Y_{i_{1} i_{2}}\right| \geqslant \tau_{n} \sqrt{n}\right) .
\end{aligned}
$$

Сначала оценим правую часть в (36). Граф из несовпадающих ребер $\widetilde{G}_{1}\left(\mathbf{i}_{s}\right)$ является деревом. Мы можем последовательно удалить все ребра и вершины графа $\widetilde{G}_{1}\left(\mathbf{i}_{s}\right)$, используя предположение (5) на каждом шаге. Мы установим справедливость оценки

$$
\frac{\tau_{n}^{2}}{n^{s}} \sum_{\mathbf{i}_{s}} \mathbb{E} Y\left(\widetilde{G}_{1}\left(\mathbf{i}_{s}\right)\right) \leqslant C^{2(s-1)} \tau_{n}^{2} .
$$

Для члена (37) имеем

$$
\begin{aligned}
\frac{1}{n^{s+1}} & \sum_{\mathbf{i}_{s}} \mathbb{E} Y\left(\widetilde{G}_{1}\left(\mathbf{i}_{s}\right)\right) \mathbb{E} Y_{i_{1} i_{2}}^{2} \mathbb{I}\left(\left|Y_{i_{1} i_{2}}\right| \geqslant \tau_{n} \sqrt{n}\right) \\
& \leqslant \frac{1}{n^{s+1}} \sum_{\mathbf{i}_{s}} \mathbb{E} Y\left(\widehat{G}_{1}\left(\widetilde{\imath}_{s}\right)\right) \sigma_{i_{2} i_{3}}^{2} \mathbb{E} Y_{i_{1} i_{2}}^{2} \mathbb{I}\left(\left|Y_{i_{1} i_{2}}\right| \geqslant \tau_{n} \sqrt{n}\right) \\
& \leqslant \frac{C^{2}}{n^{s}} \sum_{i_{1}, i_{2}=1}^{n} \mathbb{E} Y_{i_{1} i_{2}}^{2} \mathbb{I}\left(\left|Y_{i_{1} i_{2}}\right| \geqslant \tau_{n} \sqrt{n}\right) \sum_{\widehat{\imath}_{s}} \mathbb{E} Y\left(\widehat{G}_{1}\left(\widetilde{\imath}_{s}\right)\right) .
\end{aligned}
$$

Снова используя (5), можно показать, что

$$
\sum_{\widehat{\imath}_{s}} \mathbb{E} Y\left(\widehat{G}_{1}\left(\widetilde{\imath}_{s}\right)\right) \leqslant C^{2(s-2)} n^{s-2} .
$$


В силу (39) и (40) получим

$$
\frac{1}{n^{s+1}} \sum_{\mathbf{i}_{s}} \mathbb{E} Y\left(\widetilde{G}_{1}\left(\mathbf{i}_{s}\right)\right) \mathbb{E} Y_{i_{1} i_{2}}^{2} \mathbb{I}\left(\left|Y_{i_{1} i_{2}}\right| \geqslant \tau_{n} \sqrt{n}\right) \leqslant C^{2(s-1)} L_{n}\left(\tau_{n}\right) .
$$

Из (38) и (41) получим оценку

$$
\frac{1}{n^{s+1}} \sum_{\mathbf{i}_{s}} \mathbb{E} Y\left(G_{1}\left(\mathbf{i}_{s}\right)\right) \leqslant C^{2(s-1)} \tau_{n}^{2}+C^{2(s-1)} L_{n}\left(\tau_{n}\right) .
$$

Окончательно для случаев і)-іiі) будем иметь

$$
\frac{1}{n^{m+1}} \sum_{G(\mathbf{i}) \in \Gamma(2 m, t)} \mathbb{E} Y(G(\mathbf{i})) \leqslant{ }_{m} C^{2(m-1)}\left(\tau_{n}^{2}+L_{n}\left(\tau_{n}\right)\right)=o_{m}(1) .
$$

В качестве примера мы рекомендуем проверить алгоритм для графа, изображенного на рис. 2.

Легко видеть, что число канонических графов в категории $C_{3}$ имеет порядок $O_{m}(1)$. Окончательно получаем следующую оценку для $S_{3}$ :

$$
S_{3}=o_{m}(1)
$$

Нам остается оценить $S_{1}$. Для графа из категории $C_{1}$ мы знаем, что $k$ должно быть четным числом, например, $k=2 m$. Для графов из категории $C_{1}$, используя обозначения $\mathbf{i}_{t}, \widetilde{\imath}_{t}$ и $\widehat{\imath}_{t}$, будем полагать $t=m+1$.

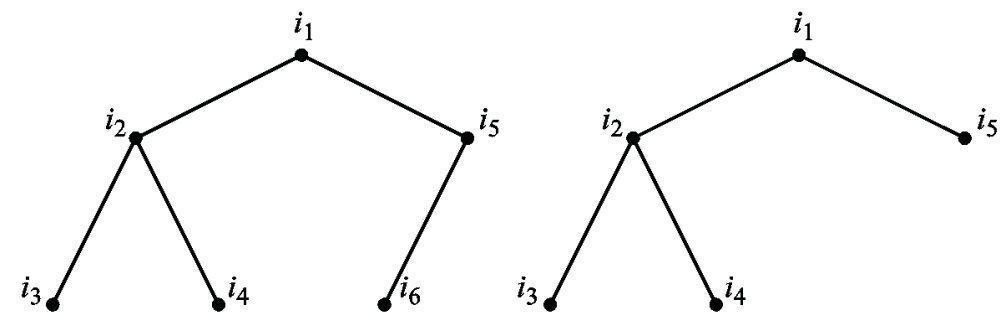

Рис. 3. Слева: дерево несовпадающих вершин графа $\Gamma_{1}(10)$. Справа: дерево несовпадающих вершин графа $\Gamma_{1}(10)$ с удаленным листом $i_{6}$.

Слева на рис. 3 изображен пример дерева несовпадающих вершин графа $\Gamma_{1}(2 m)$ для $m=5$. Член, соответствующий этому дереву, равен $Y\left(G\left(\mathbf{i}_{6}\right)\right)=Y_{i_{1} i_{2}}^{2} Y_{i_{2} i_{3}}^{2} Y_{i_{2} i_{4}}^{2} Y_{i_{1} i_{5}}^{2} Y_{i_{5} i_{6}}^{2}$.

Обозначим через $\sigma^{2}\left(\mathbf{i}_{m+1}\right)=\sigma^{2}\left(G\left(\mathbf{i}_{m+1}\right)\right)$ произведение $m$ чисел $\sigma_{i_{s} i_{t}}^{2}$, где $i_{s}, i_{t}, s<t$, - некоторые вершины графа $G\left(\mathbf{i}_{m+1}\right)$, соединенные ребром из этого графа. В нашем примере $\sigma^{2}\left(\mathbf{i}_{m+1}\right)=\sigma^{2}\left(\mathbf{i}_{6}\right)=$ $\sigma_{i_{1} i_{2}}^{2} \sigma_{i_{2} i_{3}}^{2} \sigma_{i_{2} i_{4}}^{2} \sigma_{i_{1} i_{5}}^{2} \sigma_{i_{5} i_{6}}^{2}$. 
Если $m=1$, то $\sigma^{2}\left(\mathbf{i}_{2}\right)=\sigma_{i_{1} i_{2}}^{2}$ и

$$
\frac{1}{n^{2}} \sum_{\substack{i_{1}, i_{2}=1 \\ i_{1} \neq i_{2}}}^{n} \sigma_{i_{1} i_{2}}^{2}=\frac{1}{n} \sum_{i_{1}=1}^{n}\left[\frac{1}{n} \sum_{i_{2}=1}^{n} \sigma_{i_{1} i_{2}}^{2}-1\right]+1+o(1)
$$

где мы использовали соотношение $n^{-2} \sum_{i_{1}=1}^{n} \sigma_{i_{1} i_{1}}^{2}=o(1)$. В силу (4) первое слагаемое в (42) имеет порядок $o(1)$. Число канонических графов в $C_{1}$ для $m=1$ равно 1 . Мы заключаем, что для $m=1$

$$
S_{1}=n^{-2} \sum_{\Gamma_{1}(2)} \sum_{\substack{i_{1}, i_{2}=1 \\ i_{1} \neq i_{2}}}^{n} \sigma_{i_{1} i_{2}}^{2}=1+o(1)
$$

Далее предположим, что $m>1$. Рассмотрим дерево несовпадающих ребер графа $G$. Можно найти лист в дереве, скажем, $i_{q}$, и вершину $i_{p}$, которая соединена с $i_{q}$ ребром из этого дерева. Мы имеем $\sigma^{2}\left(\mathbf{i}_{m+1}\right)=$ $\sigma^{2}\left(\widetilde{\imath}_{m+1}\right) \sigma_{i_{p} i_{q}}^{2}$, где $\sigma^{2}\left(\widetilde{\imath}_{m+1}\right)=\sigma^{2}\left(G\left(\widetilde{\imath}_{m+1}\right)\right)$.

В нашем примере можно взять лист $i_{6}$. Справа на рис. 3 изображено дерево с удаленным листом $i_{6}$. Мы имеем $\sigma_{i_{p} i_{q}}^{2}=\sigma_{i_{5} i_{6}}^{2}$ и $\sigma^{2}\left(\widetilde{\imath}_{6}\right)=\sigma_{i_{1} i_{2}}^{2} \sigma_{i_{2} i_{3}}^{2} \sigma_{i_{2} i_{4}}^{2} \sigma_{i_{1} i_{5}}^{2}$.

Легко видеть, что

$$
\begin{aligned}
\frac{1}{n^{m+1}} \sum_{\mathbf{i}_{m+1}} \sigma^{2}\left(\mathbf{i}_{m+1}\right)= & \frac{1}{n^{m+1}} \sum_{\tilde{\imath}_{m+1}} \sigma^{2}\left(\widetilde{\imath}_{m+1}\right) \sum_{i_{q}=1}^{n} \sigma_{i_{p} i_{q}}^{2}+o_{m}(1) \\
= & \frac{1}{n^{m}} \sum_{\tilde{\imath}_{m+1}} \sigma^{2}\left(\widetilde{\imath}_{m+1}\right)\left[\frac{1}{n} \sum_{i_{q}=1}^{n} \sigma_{i_{p} i_{q}}^{2}-1\right] \\
& +\frac{1}{n^{m}} \sum_{\tilde{\imath}_{m+1}} \sigma^{2}\left(\widetilde{\imath}_{m+1}\right)+o_{m}(1),
\end{aligned}
$$

где мы добавили некоторые графы из категории $C_{3}$ и воспользовались оценками, аналогичными оценкам для члена $S_{3}$. Теперь покажем, что слагаемое (43) имеет порядок $o_{m}(1)$. Заметим, что

$$
\begin{aligned}
& \frac{1}{n^{m}} \sum_{\tilde{\imath}_{m+1}} \sigma^{2}\left(\widetilde{\imath}_{m+1}\right)\left|\frac{1}{n} \sum_{i_{q}=1}^{n} \sigma_{i_{p} i_{q}}^{2}-1\right| \\
& \quad=\frac{1}{n} \sum_{i_{p}=1}^{n}\left|\frac{1}{n} \sum_{i_{q}=1}^{n} \sigma_{i_{p} i_{q}}^{2}-1\right| \frac{1}{n^{m-1}} \sum_{\hat{\imath}_{m+1}} \sigma^{2}\left(\widetilde{\imath}_{m+1}\right) .
\end{aligned}
$$

Последовательно удаляя все вершины из дерева и используя (5), получим

$$
\frac{1}{n^{m-1}} \sum_{\hat{\imath}_{m+1}} \sigma^{2}\left(\widetilde{\imath}_{m+1}\right) \leqslant C^{2(m-1)} \text {. }
$$


В силу (46) и (4) мы показали, что слагаемое (43) имеет порядок $o_{m}(1)$. Для первого слагаемого в (44) повторим процедуру и остановимся, если у нас осталось только две различные вершины. На последнем шаге мы воспользуемся результатом (42). Окончательно имеем

$$
S_{1}=\frac{1}{n^{m+1}} \sum_{\Gamma_{1}(2 m)} \sum_{\mathbf{i}_{m+1}} \sigma^{2}\left(\mathbf{i}_{m+1}\right)=\frac{1}{m+1}\left(\begin{array}{c}
2 m \\
m
\end{array}\right)+o_{m}(1),
$$

что завершает доказательство теоремы 3.

\section{СПИСОК ЛИТЕРАТУРЫ}

1. Wigner E.P. On the distribution of the roots of certain symmetric matrices. - Ann. Math., 1958, v. 67, p. 325-327.

2. Arnold L. On Wigner's semicircle law for the eigenvalues of random matrices. - Z . Wahrscheinlichkeitstheor. verw. Geb., 1971, v. 19, p. 191-198.

3. Пастур Л. А. Спектры случайных самосопряженных операторов. - Успехи матем. наук, 1973, т. 28, № 1, с. 3-64.

4. Shlyakhtenko D. Random gaussian band matrices and freeness with amalgamation. Int. Math. Res. Not., 1996, №20, p. 1013-1025.

5. Erdös L. Universality of Wigner random matrices: a survey of recent results. arXiv:1004.0861.

6. Götze F., Tikhomirov A.N. Limit theorems for spectra of random matrices with martingale structure. - Теория вероятн. и ее примен., 2006, т. 51, в. 1, с. 171-192.

7. Hall P., Heyde C.C. Martingale Limit Theory and Its Application. New York: Academic Press, 1980, 308 p.

8. Rosenzweig N. Statistical Mechanics of Equally Likely Quantum Systems. New York: Benjamin, 1963, $91 \mathrm{p}$.

9. Bronk B.V. Topics in the theory of random matrices. $\mathrm{PhD}$ thesis. Princeton: Princeton Univ., 1964.

10. Наумов A. А. Предельные теоремы для двух классов случайных матриц с гауссовскими элементами. - Зап. науч. сем. ПОМИ, 2013, т. 412, с. 214-225.

11. Wishart J. Generalised product moment distribution in samples from a normal multivariate population. - Biometrika, 1928, v. 20, p. 32-52.

12. Марченко В. А., Пастур Л. А. Распределение собственных значений в некоторых ансамблях случайных матриц. - Матем. сб., 1967, т. 72, № 4, с. 507-536.

13. O'Rourke $S$. A note on the Marchenko-Pastur law for a class of random matrices with dependent entries. - Electron. Commun. Probab., 2012, v. 17, paper № 28, 13 p.

14. Götze F., Tikhomirov A. N. Limit theorems for spectra of positive random matrices under dependence. - Зап. науч. сем. ПОМИ, 2004, т. 311, с. 92-123.

15. Adamczak R. On the Marchenko-Pastur and circular laws for some classes of random matrices with dependent entries. - Electron. J. Probab., 2011, v. 16, № 37, p. 10681095.

16. Бенткус B. Новый подход к аппроксимациям в теории вероятностей и теории операторов. - Liet. Mat. Rink., 2003, v. 43, № 4, p. 444-470.

17. Pastur L., Shcherbina M. Eigenvalue Distribution of Large Random Matrices. Providence: Amer. Math. Soc., 2011, 632 p.

18. Bai Z., Silverstein J. W. Spectral Analysis of Large Dimensional Random Matrices. Dordrecht: Springer, 2010, $551 \mathrm{p}$. 